\title{
miR-29a Promotes the Neurite Outgrowth of Rat Neural Stem Cells by Targeting Extracellular Matrix to Repair Brain Injury
}

\author{
Rongjie Ma, ${ }^{1}$ Min Wang, ${ }^{2,3}$ Shane Gao, ${ }^{4}$ Liang Zhu, ${ }^{4}$ Liming Yu, ${ }^{5}$ Daiyu Hu, ${ }^{6,7}$ Luying Zhu, Wei Huang, ${ }^{5}$ \\ Weihua Zhang, ${ }^{5}$ Jiajia Deng, ${ }^{5}$ Jie Pan, ${ }^{5}$ Hua He, ${ }^{8}$ Zhengliang Gao, ${ }^{6,7, *}$ Jun $\mathrm{Xu}^{4, *}$ and Xinxin Han ${ }^{5, *}$
}

\begin{abstract}
Neural stem cells (NSCs) can generate new neurons to repair brain injury and central nervous system disease by promoting neural regeneration. MicroRNAs (miRNAs) involve in neural development, brain damage, and neurological diseases repair. Recent reports show that several miRNAs express in NSCs and are important to neurogenesis. Neurites play a key role in NSC-related neurogenesis. However, the mechanism of NSC neurite generation is rarely studied. We surprisingly noticed that the neurites increased after bone morphogenetic protein (BMP) treatment in rat NSCs. This process was accompanied by the dynamic change of miRNA-29. Then we discovered that miR-29a regulated neural neurites in rat hippocampus NSCs. Overexpression of miR29a reduced the cell soma area and promoted the neurite outgrowth of NSCs. Cell soma area became small, whereas the number of neurite increased. Moreover, neurite complexity increased dramatically, with more primary and secondary branches after miR-29a overexpression. In addition, miR-29a overexpression still maintained the stemness of NSCs. Besides, we identified that miR-29a can promote the neurite outgrowth by targeting extracellular matrix-related genes like Fibrillin 1 (Fbnl), Follistatin-like 1 (Fstll), and laminin subunit gamma 2 (Lamc2). These findings may provide a novel role of miR-29a to regulate neurite outgrowth and development of NSCs. We also offered a possible theoretical basis to the migration mechanism of NSCs in brain development and damage repair.
\end{abstract}

Keywords: miR-29, neural stem cell, brain injury, neurite, BMP, extracellular matrix

\section{Introduction}

$\mathrm{N}$ EURAL STEM CELLS (NSCs) have self-renewing ability and can differentiate into neurons, astrocytes, and oligodendrocytes in mammalian brain [1]. They are important to neuronal plasticity, neurogenesis, and neuronal integration [2,3]. Neurogenesis initiates from NSCs and produces new functional neurons. Therefore, neurogenesis plays an important role both in embryonic neural development and adult brain plasticity [4,5]. In adult brain, neurogenesis is regulated by several key factors and signaling mechanisms [5].

MicroRNAs (miRNAs) regulate gene expression at the post-translational levels by either inhibiting messenger RNA (mRNA) translation or inducing mRNA degradation in many biological processes [6,7]. miRNAs are enriched in central nervous system and play pivotal roles during neurogenesis and brain development [8,9]. Reports show that miRNAs involve in neurogenesis, cell fate determination, and cell migration [10]. For example, miR-9 regulates NSC proliferation and differentiation by targeting nuclear receptor TLX (nuclear receptor subfamily 2 group E member 1, NR2E1) and other transcription factors $[11,12]$. The interplay between epigenetic machinery and miRNAs associates with several cellular mechanism of NSCs fate determination [13].

A key step of neurogenesis is neuronal maturation, which includes dendritic and axonal growth, synaptogenesis, neuronal and synaptic pruning, and so on [14,15]. miRNAs have been implicated in neuronal maturation [16]. miR-9 contributes to

\footnotetext{
${ }^{1}$ Department of Gynecology and Obstetrics, Shanghai Tenth People's Hospital, Tongji University School of Medicine, Shanghai, China.

${ }^{2}$ School of Medicine, Jiaxing University, Jiaxing, China.

${ }^{3}$ Oral Biomedical Engineering Laboratory, Shanghai Stomatological Hospital, Fudan University, Shanghai, China.

${ }^{4}$ East Hospital, Tongji University School of Medicine, Shanghai, China.

${ }^{5}$ Oral Biomedical Engineering Laboratory, Shanghai Stomatological Hospital, Fudan University, Shanghai, China.

${ }^{6}$ Shanghai Tenth People's Hospital, Tongji University School of Medicine, Shanghai, China.

${ }^{7}$ Lifeng Institute of Regenerative Medicine, Tongji University, Shanghai, China.

${ }^{8}$ Department of Neurosurgery, Third Affiliated Hospital of Second Military Medical University, Shanghai, China.

*These authors are considered senior authors.
} 
neural cells and regulates NSC proliferation and differentiation. In addition, miR-9 overexpression promotes axonal branching and reduces the axonal outgrowth [17]. miR-21 exhibits neurites outgrowth [18]. miR-431 promotes axonal outgrowth and induces axonal regeneration [19]. Not only axons, miRNAs also play an important role in dendrites. miR-137 regulates the dendritic morphology by targeting a ubiquitin ligase named Mind bomb one (Mib1) [16]. Overexpression of miR-541 represses synapsin-I and reduces the neurite extensions [20]. miR-132 is reported to promote dendritic growth and remodeling [21]. These findings illustrate the important role of miRNAs in neurite outgrowth and branching.

Extracellular matrix (ECM) is a complex network of material such as proteins and polysaccharides, provides structural, adhesive, and biochemical signaling support to cells. The ECM is composed of three molecule types: (1) structural proteins, that is, glycoproteic collagens (Col) and nonglycosylated proteins elastins (Eln); (2) specialized glycoproteins, such as fibrillin (Fbn), fibronectin (FN), and laminin (Lam); and (3) heavily glycosylated proteoglycans $(\mathrm{PoG})$, such as chondroitin, heparan, keratan sulfate proteoglycans, and hyaluronic acid [22]. Signaling from the ECM influences regulation of gene expression [22]. Cell surface receptors transduce signals into cells from ECM, which regulate diverse cellular functions, such as survival, growth, migration, and differentiation [23].

miR-29 family suppress many ECM genes, including collagens, elastin, and fibrillins [24,25]. Family members' miR-29a/29b/29c share the same seed sequence, and they are regulated by various transcriptional regulators and signaling pathways [26]. They have emerged as a key modulator of ECM homeostasis [27]. miR-29b overexpression is associated with aging vasculature [24] and aneurysm development $[28,29]$. Reduced miR-29b is noted in pulmonary fibrosis by upregulation of ECM genes [30]. miR-29 inhibition enhances elastin expression with elastin haploinsufficiency [31]. miR-29a has been demonstrated the anti-tumor functions as a tumor suppressor in chronic lymphocytic leukemia and lung cancer [32], hepatocellular carcinoma [33], pancreatic cancer cells [34], pediatric acute myeloid leukemia [35], papillary thyroid carcinoma [36], and myeloma [37]. However, miR-29a also promotes tumorigenesis in breast cancer [38], nasopharyngeal carcinoma [27], and oral squamous cell carcinoma [39]. miR-29a also induces apoptosis in a p53-dependent way [40]. Thus, miR-29a might have different functions in different cells.

Moreover, ECM regulation by miR-29a has been implicated in fibrosis of many organs including heart $[41,42]$ and liver $[43,44]$. Reports point that miR-29a highly expresses in mice cerebrum, and can trigger upregulation axon branching by directly repressing doublecortin (DCX) [45-47]. miR-29a also increases neurite outgrowth by decreasing PTEN (phosphatase and tensin homolog) expression in PC12 cells [48]. Yesassociated protein (YAP) promotes the neurite outgrowth by targeting the promoter of miR-29a in N2a cells [49].

During the maturation of miRNAs, the pre-miRNA hairpin is cleaved to generate two strands. In most miRNAs, the more stable strand is functional and less stable strand tends to degradation. Only one strand is overly dominant, although in some cases, both strands could be functional [6]. Based on miRBase analysis (www.mirbase.org), we found that miR-29a-3p was more abundant between two strands of miR-29a. Moreover, miR-29a-3p potentially targeted large number of ECM genes as predicting by TargetScan (www.targetscan.org). These findings offer some inspirations and motivate us to delve into the role of miRNA-29a in NSCs. However, the mechanism of NSC neurite generation is rarely reported.

In this study, we observed that neurites of rat NSCs increased after bone morphogenetic protein (BMP) treatment. This process was accompanied by the dynamic change of miRNA-29a. Then we investigated the function of miR-29a in this process. We showed that overexpression of miR-29a promoted the neurite outgrowth of NSCs. Moreover, neurite complexity increased dramatically, with multiple primary and secondary branches. Then we found that miR-29a significantly influenced many ECM-related genes, including the collagen family gene Col3al (collagen, type III, alpha 1); the laminin-encoding gene laminin subunit gamma 2 (Lamc2); the fibrillin family gene $F b n 1$, Sparc (secreted acidic cysteine rich glycoprotein), and Follistatin-like 1 (Fstl1). Similar neurite outgrowth displayed after several typical ECM genes knock down. Together, our results suggested that miR-29a may play an important role in NSC neurite outgrowth and development by targeting ECM.

\section{Materials and Methods}

\section{Cell culture}

Rat NSC cells were cultured under the following conditions: Dulbecco's modified Eagle's medium (DMEM)/ F12 (Gibco, Grand Island, NY) containing $1 \times \mathrm{N} 2$ supplement (Gibco), $1 \times$ GlutaMAX (Gibco), $1 \times$ penicillin/streptomycin (Gibco), and $20 \mathrm{ng} / \mathrm{mL}$ FGF-2 (HumanZyme, Chicago, IL). When cell density was up to $70 \%-80 \%$, we prepared to passage. Trypsin (0.05\%; Gibco) was used to digest NSCs, and trypsin inhibitor (Gibco) to stop the trypsin. Then cells were seeded at the appropriate density after counting.

\section{Poly-L-ornithine and laminin-coated plates}

The plates were coated with poly-L-ornithine (Sigma, St. Louis, MO) and laminin (Thermo Fisher, Waltham, MA) for rat NSCs culture and passage. The plates were treated by $0.5 \mu \mathrm{g} / \mathrm{mL}$ poly-L-ornithine (dissolving in water) at room temperature for $16 \mathrm{~h}$, and then washed with phosphatebuffered saline (PBS). Finally, $5 \mu \mathrm{g} / \mathrm{mL}$ laminin was added into the plates for at least $16 \mathrm{~h}$. The coated plates can be stored at $-20^{\circ} \mathrm{C}$ and thaw them before use. The coverslips should also be coated following the same method (if coverslip is needed).

\section{BMP-treated NSC cells}

The final concentration of BMP-4 (HumanZyme) was $50 \mathrm{ng} / \mathrm{mL}$. BMP-4 was added to the culture medium after passage. We did not change medium or passage during treatment. Discard BMP-4 according to the required time points.

\section{NSC cells transfection}

The miR-29a precursor sequences were cloned into FG12 vector (No. 14884; Addgene) to construct recombinant miR29a overexpression plasmid (miR-29 OX), with scrambled 
miRNA control plasmid (Control). After $48 \mathrm{~h}$ of BMP-4 treatment, the transfection of plasmids was performed in NSCs using electroporation following the manufacturer's instructions (Pulse Generator CUY21EDIT II; BEX), and the concentration of plasmids was $1 \mu \mathrm{g} / \mu \mathrm{L}$. After transfection, NSCs were seeded into six-well plates at a density of $1.5 \times 10^{5} /$ well. For reverse transcription-quantitative real time polymerase chain reaction (RT-qPCR) assay and RNA sequencing (RNA-Seq), NSCs were treated by $50 \mathrm{ng} / \mathrm{mL}$ BMP-4 for $48 \mathrm{~h}$, then miR-29a-3p mimics and mimics control were transfected into NSCs by Lipofectamine 3000 (Invitrogen, Carlsbad, CA), respectively, according to the instructions. miR-29a-3p mimics were double-stranded small RNA molecules, designed based on the mature sequence of rat miR-29a$3 p$, synthesized by GenePharma Co. (Shanghai, China). For gene knockdown experiments, specific small interfering RNA (siRNA) and control were transfected into NSCs by Lipofectamine 3000 using the same method. Each set of experiments was repeated at least three times.

\section{$R T-q P C R$ assay}

Total RNA was isolated from NSCs using TRIzol reagent (Invitrogen). miR-29 and mRNAs were reversely transcribed into complementary DNA (cDNA) using TaqMan ${ }^{\mathrm{TM}}$ Advanced miRNA cDNA Synthesis Kit (Thermo Fisher) or Reverse Transcription System Kit (Takara, Dalian, China). RT-qPCR reactions were conducted using RT-qPCR (SYBR Green) Kit (Tiangen, Beijing, China) on an Applied Biosystems 7500 Real-time PCR System (Applied Biosystems, Foster City, CA). All samples were amplified in technical triplicates. The relative quantifications of miR-29 and interested mRNAs were performed by the $2^{-\Delta \Delta \mathrm{Ct}}$ method. U6 and GAPDH were used as endogenous controls to normalize the data of miR-29 and mRNAs, respectively. The primers of interested mRNAs were as follows: Sox2 [SRY (sex determining region Y)-box transcription factor 2] forward primer: 5'-CGGCGGCAACCAGAAGAACAG-3', reverse primer: 5'-CGCTTGGCCTCGTCGATGAAC-3'; Nes (Nestin) forward primer: 5'-ATGAGGAAGGAGCAGAGTCAGG AG-3', reverse primer: 5'-CAGCACCTCTCAAGCCATCAT CC-3'; Fstll forward primer: 5'-GTGGCAGTAATGGCAA GACCTACC-3', reverse primer: 5'-CGGTTAGCCTGATA GCAGACAACG; Col3al forward primer: 5'-GACACGC TGGTGCTCAAGGAC, reverse primer: 5'-GTTCGCCTGAA GGACCTCGTTG; Lamc2 forward primer: 5'-AGACAC GCTCAACACATTGGAAGG-3', reverse primer: 5'-AGGT GGAGGTGGTTGCTCTGTC-3'; Sparc forward primer: 5'AAGCTCCACCTGGACTACATCGG-3', reverse primer: 5'-CGCTTCTCGTTCTCGTGGATCTTC-3'; Fbnl forward primer: 5'-TGCCGCATATCTCCTGACCTCTG-3', reverse primer: 5'-TAGCCTTCGTCACACTTGCATTCG-3'; Cdk6 (cyclin-dependent kinase 6) forward primer: 5'-TGACA GACATCGACGAGCTAGGC-3', reverse primer: 5'-GCTG GACGACAGGTGAGAATGC-3'; Mmp24 (matrix metallopeptidase 24) forward primer: 5'-GAGGTAGAGCGG CGGAAGGAG-3', reverse primer: 5'-CCACAGCCACAG CATTCACAGAG-3'; Col5a3 (Collagen, type V, alpha 3) forward primer: 5'-TCAGGTGACCACAGGCACTCT ATC-3', reverse primer: 5'-TTGATGGTGGCTGCTGTTG TCTG-3'. For gene knockdown experiments, we also used RT-qPCR to quantify silencing efficiency. Methods and primers were as described previously. All primers were synthesized by Sangon Biotech Co. (Shanghai, China).

\section{Immunostaining assay}

Cells were fixed with $4 \%$ PFA for $15 \mathrm{~min}$ at room temperature, and then washed with PBS for $3 \times 10 \mathrm{~min}$. After blocking with $3 \%$ bovine serum albumin (BSA) solution in PBS containing $0.3 \%$ Triton $\mathrm{X}-100$ and $0.05 \%$ Tween-20 at room temperature for $1 \mathrm{~h}$, cells were incubated with primary antibodies at $4{ }^{\circ} \mathrm{C}$ overnight. The dilution buffer of antibodies was the same as blocking solution and the dilution ratios were as follows: Sox2 (1:2,000; Santa Cruz, Dallas, TX), neuroepithelial stem cell protein Nestin (Nes; 1:2,000; Abcam, Cambridge, MA), marker of proliferation Ki67 (1:1,000; Thermo Fisher), DCX (1:500; Santa Cruz), glial fibrillary acidic protein (GFAP, 1:1,000; DakoCytomation, Carpinteria, CA), and microtubule-associated protein 2 (MAP2, 1:1,000; Synaptic Systems, Gottingen, Germany). On the second day, primary antibodies were discarded and cells were washed with PBS containing $0.05 \%$ Tween-20 for $3 \times 10 \mathrm{~min}$. Thereafter, cells were incubated with second antibodies at room temperature for $2 \mathrm{~h}$. The dilution ratio of fluorescent-labeled second antibodies (Jackson ImmunoResearch Laboratories, West Grove, PA) was 1:1,000. Finally, nuclei were stained with DAPI (1:1,000; Sigma) for 10 min. Then the solution was discarded and cells were washed with PBS containing $0.05 \%$ Tween-20 for $3 \times 10 \mathrm{~min}$. If coverslips were used, the coverslips were picked up carefully and sealed with mounting media (Thermo Fisher).

\section{RNA sequencing}

NSCs were plated in $100-\mathrm{mm}$ plates and treated with $50 \mathrm{ng} / \mathrm{mL}$ BMP-4 for $48 \mathrm{~h}$. Then we transfected cells with miR-29a mimics and control, respectively, by Lipofectamine 3000 according to the instructions. After 48-h transfection (up in BMP conditions), RNA was isolated, and then total RNA was treated by mRNA enrichment or rRNA removal. Purified mRNA was fragmented into small pieces, and then first-strand cDNA was generated using random hexamerprimed reverse transcription, followed by a second-strand cDNA synthesis. Afterward, A-Tailing Mix and RNA Index Adapters were added by incubating to end repair. The cDNA fragments obtained from previous step were amplified by PCR, and products were purified. Then the double-stranded PCR products were heated, denatured, and circularized by the splint oligo sequence to get the final library. This singlestrand circle DNA was sequenced on BGIseq500 platform (BGI Co.). Sample processing and sequencing were performed by BGI Co. in three separate experiments.

The raw data obtained by sequencing were called raw reads. Low-quality reads was filtered out and the so-called clean reads were then aligned to the reference genome by HISAT (Hierarchical Indexing for Spliced Alignment of Transcripts) Software [50], following by new transcript prediction, single nucleotide polymorphism (SNP) and insertion-deletion (InDel), and differential splicing gene detection. After obtaining a new transcript, the new transcript was added with potential coding proteins to the reference gene sequence to form a complete reference sequence for alignment using Bowtie2 [51], and then calculated the gene 
and transcript expression level by RSEM [52]. Finally, differentially expressed genes between different samples were detected according to requirements. In addition, cluster analysis and functional enrichment analysis can perform on the differentially expressed genes.

\section{Gene ontology enrichment and pathway analysis}

We performed pathway and network analysis of differentially expressed genes in Metascape (http://metascape.org). For the given gene list, pathway and process enrichment analysis were carried out with the following ontology sources: GO Biological Processes, KEGG Pathway, Reactome Gene Sets, and CORUM. All genes in the genome had been used as the enrichment background. Terms with a $P<0.01$, a minimum count of 3 , and an enrichment factor $>1.5$ (the enrichment factor is the ratio between the observed counts and the counts expected by chance) were collected and grouped into clusters based on their membership similarities. More specifically, $P$ values were calculated based on the accumulative hypergeometric distribution, and $\mathrm{q}$ values were calculated using the Banjamini-Hochberg procedure to account for multiple testings. Kappa scores were used as the similarity metric when performing hierarchical clustering on the enriched terms, and subtrees with a similarity of $>0.3$ were considered a cluster. The most statistically significant term within a cluster was chosen to represent the cluster.

\section{Sholl analysis}

Sholl analysis was performed by a plugin for ImageJ. It creates a series of concentric circles around the cell soma, and counts how many times the cell intersects with the circumference of these circles $[53,54]$. User guide provided by Tiago Ferreira and Wayne Rasband of ImageJ (https://imagej.nih.gov/ ij/docs/guide/146.html) were followed and the analysis parameters were set as given hereunder: starting radius: $10 \mu \mathrm{m}$, radius step size: $10 \mu \mathrm{m}$. Then we calculated the sum intersections of cells and the mean intersections for each circle.

\section{Statistical analysis}

Images were taken under the inverted fluorescence microscope (ECLIPSE Ti; Nikon) or confocal microscope (TCS SP5 II; Leica). Cells were quantified blind using the ImageJ software. Then, the data were collected from ImageJ and calculated by Excel or SPSS. Data were given as mean \pm standard deviation (SD). Error bars were defined as SD. Statistical comparison of data sets was performed by two-tailed Student's $t$-test or one-way analysis of variance (ANOVA). $t$-Tests were used in Figs. 5 and 6. ANOVA was used in Figs. 3, 4, and 7. $P<0.05$ was considered to indicate significant difference. Charts were organized by Adobe Illustrator CS5.

\section{Results}

\section{Identification of rat hippocampus NSCs after BMP treatment}

NSCs were derived from adult rat hippocampus SGZ (subgranular zone). NSCs were given as a gift from Dr. Fred
H. Gage, Salk Institute. In the presence or absence of BMP, NSCs presented two different morphologies. After $50 \mathrm{ng} / \mathrm{mL}$ BMP-4 treatment for $48 \mathrm{~h}$, we were very surprised to find an increase in protrusions from NSC cell body, with more neurites and more interaction between neighboring cells compared with control (Fig. 1A).

To identify these NSC cells with more neurites, we detected them by Sox 2 and Nestin, two classic NSC cell markers. More than $90 \%$ cells were positive for Sox 2 and Nestin (Fig. 1B). This suggested that they were NSCs.

To continuously observe these NSC cells with more neurites, we selected different time points for photographing; 12, 36, 72, and $96 \mathrm{~h}$ were arranged after BMP-4 treatment. During the first $12 \mathrm{~h}$, the neurites begin to form and cell-cell connection started to establish. In the following few days, the neurites continued to grow and became more complicated (Fig. 1C, upper). Intercellular connections were also getting closer (Fig. 1C, upper). At $96 \mathrm{~h}$, we discarded the old medium and changed fresh medium without BMP-4. Similarly, we selected a series of time points to observe the morphology of NSCs after BMP discarding. The neurite growth was almost stopped and some of the protrusions began to degrade over time (Fig. 1C, lower).

\section{Dynamic change of miR-29 in NSCs}

The growth and disappearance of neurites involved in large-scale ECM synthesis and degradation. Multiple genes and signaling pathways were related to this biological process. miR-29 was a known ECM-associated miRNA, which targeted a large number of ECM-related genes [55]. To investigate whether miR-29 involved in this neurite outgrowth process, we selected the corresponding time points after $50 \mathrm{ng} / \mathrm{mL}$ BMP-4 treatment and examined the expression pattern of miR-29 in rat hippocampus NSCs. Our results demonstrated that the expression level of miR-29-3p in NSCs was higher than $5 p$ (Fig. 2). Therefore, we decided to put more attention on $3 p$ and chose miR-29-3p as the representative of miR-29 family members. miR-29-3p and 5p expressions showed a similar trend after BMP treatment. During the first $12 \mathrm{~h}$ (B0-B12), the expression of miR-29 decreased sharply, $\sim 60 \%$ down (Fig. 2). Consistent with this process, the protrusions of NSC cells began to form. After the initial decline, miR-29 expression slightly rebound. Later, the tendency began to decline again after reaching another high point in $\sim 48 \mathrm{~h}$ (B48). In $96 \mathrm{~h}$, we discarded the old medium and changed the new medium without BMP (day 0). Then miR-29 expression stopped the downward trend and began to gradually rebound (Fig. 2).

\section{Overexpression of miR-29a significantly altered the cell soma area of rat NSCs}

To further study the effect of miR-29 family to rat NSCs, we constructed pre-miR-29a overexpression plasmid and a control plasmid, then transfected them into BMP-treated NSCs separately by electroporation. On the first day after electroporation (24h), NSCs began to express green fluorescence in both the overexpression group and the control group; on the third day $(72 \mathrm{~h})$, the luminescence intensity of GFP reached a peak; but after day 5, the expression of fluorescence had a decreasing trend because it was transient 

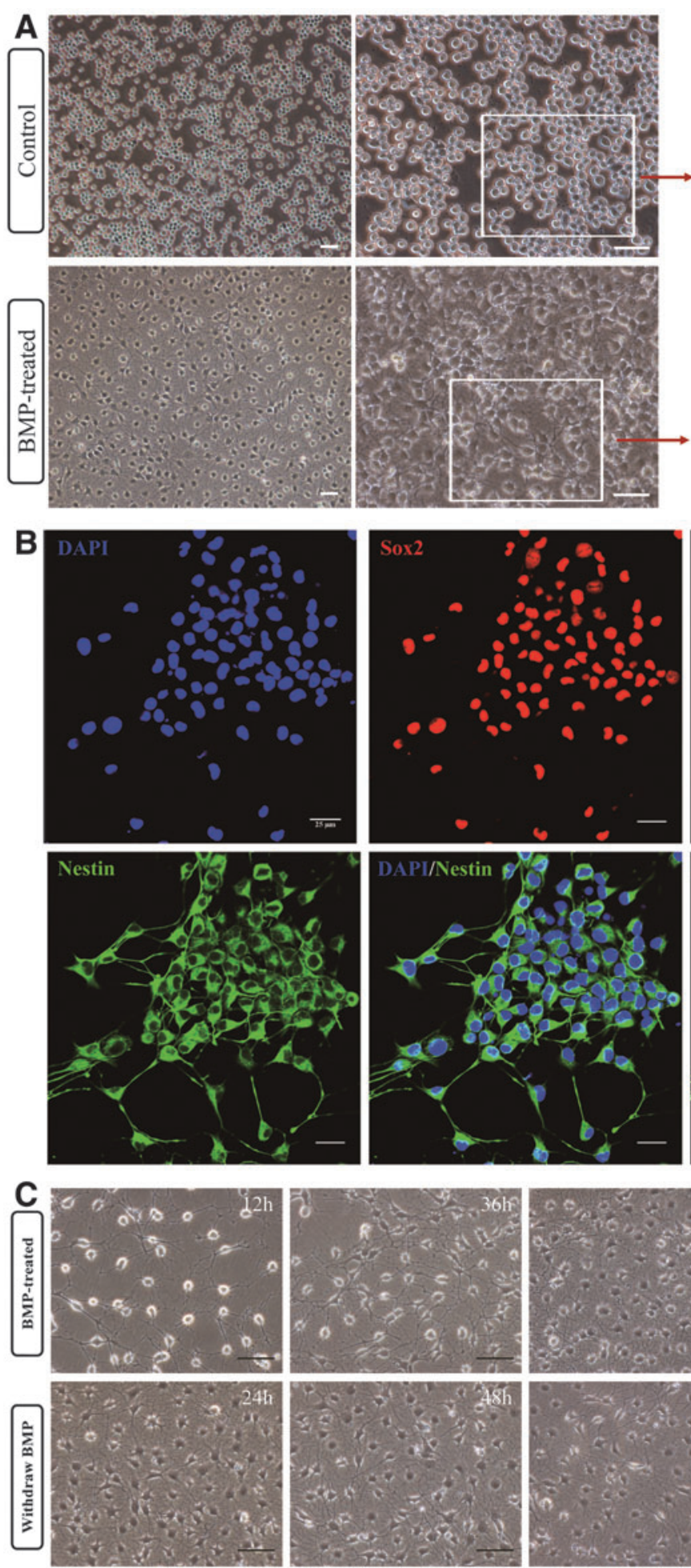
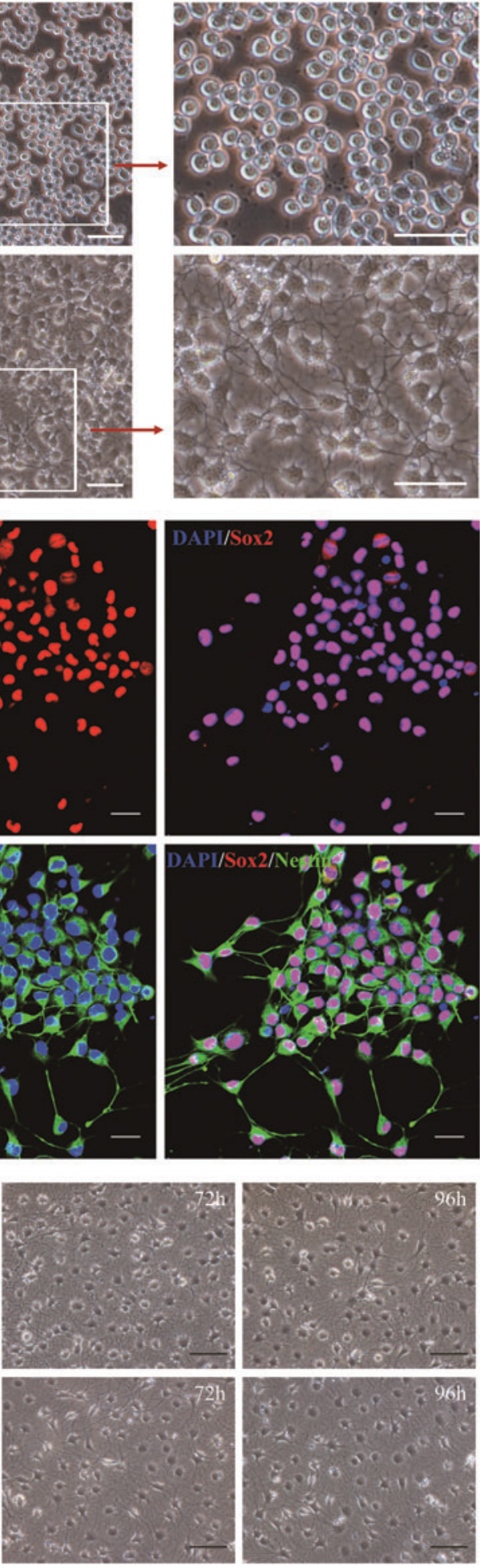

FIG. 1. Rat hippocampus NSCs after BMP treatment. (A) Morphology of normal rat hippocampus NSCs (upper) and BMP-treated NSCs (lower). (B) Rat hippocampus NSCs still expressed NSC marker Sox 2 and Nestin after BMP treatment. (C) Dynamic process of cell morphology along the time after BMP treatment (upper) and BMP withdrawn (lower). Scale bar: $50 \mu \mathrm{m}$ for $(\mathbf{A}, \mathbf{C}) ; 25 \mu \mathrm{m}$ for (B). BMP, bone morphogenetic protein; NSCs, neural stem cells; Sox2, SRY (sexdetermining region Y)-box transcription factor 2 . transfection. Some cells died and cell density also decreased (Fig. 3A). After careful comparison of GFP-positive cells, it was found that cell soma areas in the overexpression group were significantly smaller than those in the control group (Fig. 3A). We calculated the fluorescence pixels of the cell body using ImageJ, and compared the relative size of the cell soma area by using this unit. After counting thousands of cells continuously from the first day to the sixth day after electroporation, results indicated that the cell soma areas in miR-29a overexpression group were significantly reduced (Fig. 3B). In addition, the nucleoli of miR-29a overexpressed NSCs were shrunk, rounder, and smoother (Fig. 3A). 


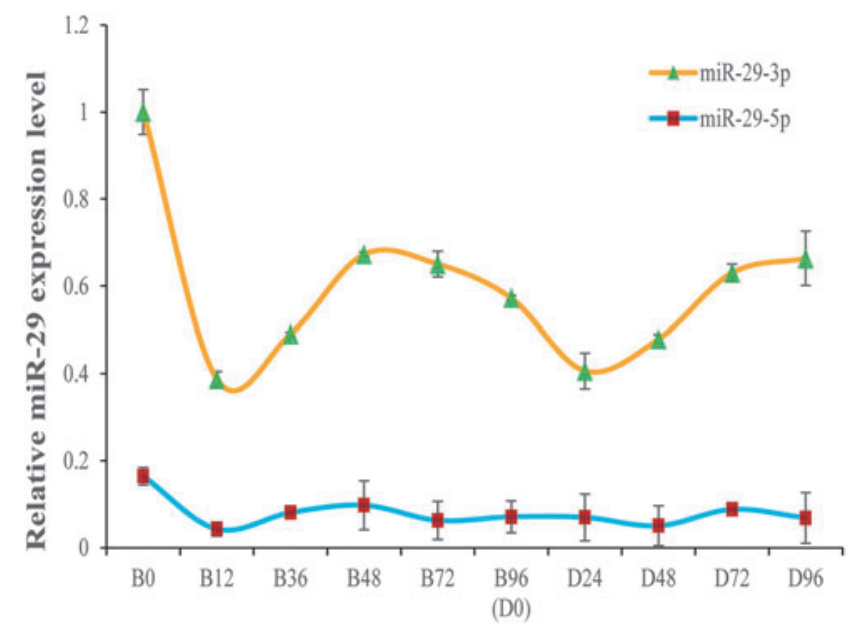

FIG. 2. Expression pattern of miR-29 after BMP treatment in rat hippocampus NSCs. Expression pattern of miR$29-3 p$ and $5 p$ during the dynamic process of BMP treatment or BMP withdrawn. Data were shown as mean \pm SD. SD, standard deviation.

\section{Effects of miR-29a on neurite outgrowth and complexity of rat NSCS}

Overexpression of miR-29a not only reduced the cell soma area, but also increased the number of cell processes. Moreover, neighboring cells were widely linked and connected (Fig. 4A). Neurite morphology was often analyzed by Sholl analysis and by counting the number of neurites and branches [56]. Therefore, we investigated the number and complexity of neurites. $\mathrm{GFP}^{+}$cells were randomly selected from the second day to the fourth day after electroporation. Then we quantified the number of processes that extended directly from the cell soma, and branches extended from these processes, including primary and secondary branches. The primary and secondary branches represented the complexity of neurites. Processes that extended directly from the cell soma of overexpressed miR-29a NSCs were greater than the control group, and this difference became more obvious over time (day 2, 5.13 vs. 3.62; day 3, 7.11 vs. 4.36; day 4, 8.26 vs. 4.97) (Fig. 4B).

Statistical results verified that the complexity of neurites in the overexpression group was much higher. On the second day, the average primary branch of miR-29a overexpressed
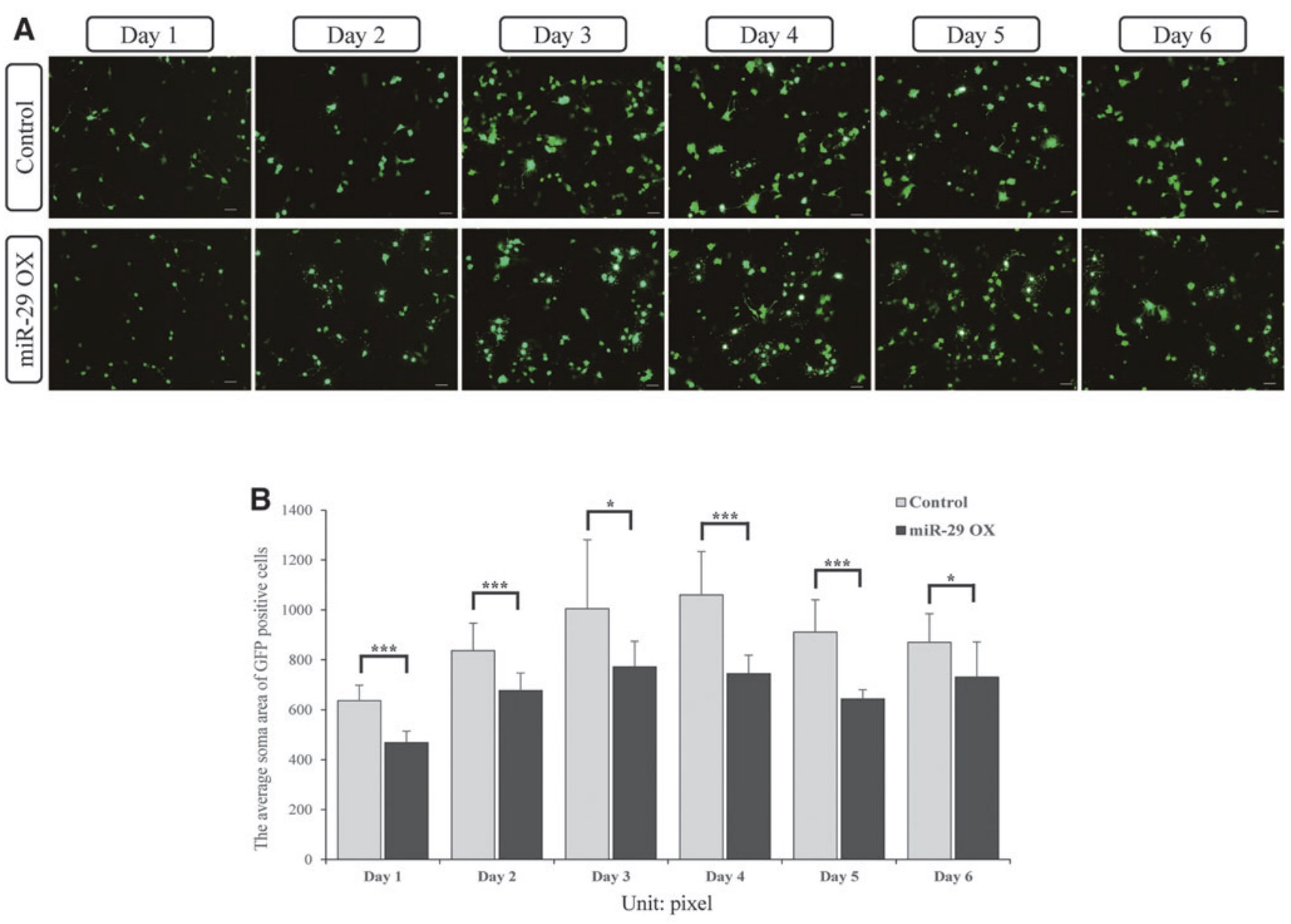

FIG. 3. Overexpression of miR-29a altered the cell soma area in rat NSCs. (A) GFP expression and cell morphology of transfected cells over days. Upper: control plasmid transfected; lower: miR-29a overexpression plasmid transfected. (B) Statistical results showed that miR-29a overexpression reduced cell soma area of NSCs. Unit: pixel. Scale bar: $50 \mu \mathrm{m}$. Data are given as mean $\pm \mathrm{SD} ; * P<0.05, * * * P<0.001$. 

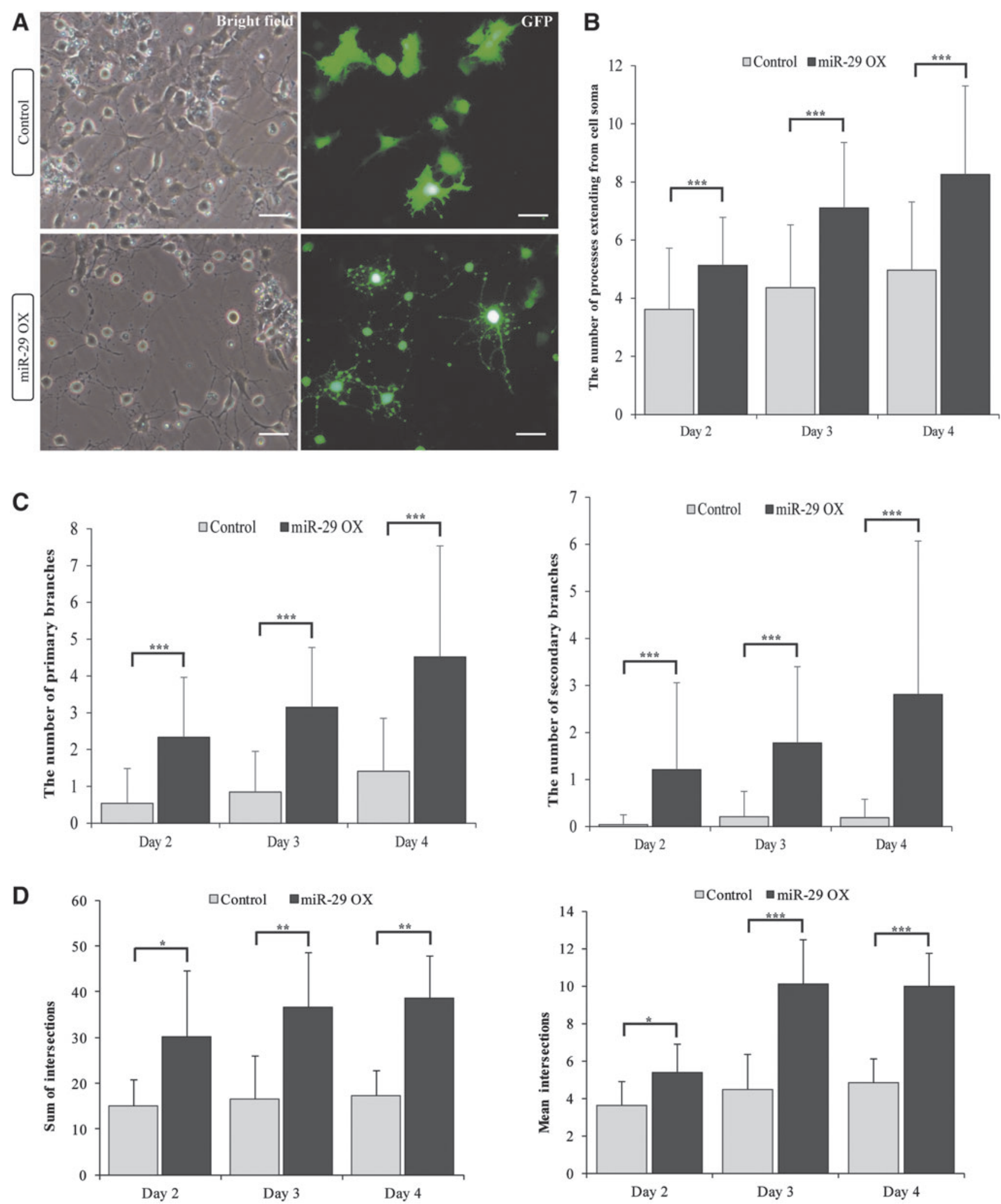

FIG. 4. miR-29a overexpression promoted neurite outgrowth and branching of rat NSCs. (A) Details of transfected cells. Upper: control plasmid transfected; lower: miR-29a overexpression plasmid transfected. (B) Statistical results showed that miR-29a overexpression increased neurite number of NSCs. (C) Neurite complexity of miR-29a overexpressed NSCs was significant increased, with more primary (left) and secondary branches (right). (D) Sholl analysis also demonstrated that the neurite complexity of miR-29a overexpressed NSCs was higher than control group, which reflected more sum intersections (left) and mean intersections (right). Scale bar: $50 \mu \mathrm{m}$. Data are given as mean $\pm \mathrm{SD} ; * P<0.05, * * P<0.01, * * * P<0.001$. 
NSCs was 2.34 , and the secondary branch was 1.21 , whereas the control group had only 0.54 primary branches and almost had no secondary branches (average 0.044). On the third day, the primary and secondary branches in the overexpressed group were 3.15 and 1.78 , respectively, which were significantly higher than the previous day. Although the control group was also increased, the average number was still very few, with only 0.85 primary branches and 0.21 secondary branches per cell on average. On the fourth day after overexpression of miR-29a, the average number of branches per cell was almost double compared with the second day: 4.52 primary branches and 2.81 secondary branches, whereas the control group had 1.41 primary branches and 0.19 secondary branches (Fig. 4C).

Sholl analysis results also showed the comparison of complexity in another perspective. Both the sum and mean intersections that overexpressed miR-29a cells were more than that of the control. On day 2, the sum intersections between neurites of miR-29a overexpressed NSCs and the concentric circles were 30.1, the mean intersections of each circumference were 5.39; whereas the sum intersections were 15 and mean intersections were 3.59 in the control group. On day 3, the sum intersections of overexpression group were 36.6 and in the control group it was 16.7, whereas the mean intersections were 10.09 and 4.45 in the two groups, respectively. The same situation also appeared on the fourth day: the overexpression group had 38.7 sum intersections and 9.97 mean intersections, which were more than that of the control group (17.3 sum intersections and 4.84 mean intersections) (Fig. 4D). Taken together, we demonstrated that miR-29a overexpression significantly increased neurite outgrowth and complexity.

\section{miR-29a overexpression maintains the characteristics of NSCs}

To confirm the characteristics of transfected cells, we detected the expression of Sox 2 and Nestin. We found that both Sox 2 and Nestin were expressed in NSC cells that transfected with either the miR-29a overexpression plasmid (Fig. 5A) or the control plasmid (Fig. 5B). It also demonstrated that overexpression of miR-29a did not alter the stem cell characteristics of NSCs.

Next, we selected several morphological markers to further confirm the effects of miR-29a overexpression, including DCX, GFAP, and MAP2. Both miR-29a overexpression and control NSC cells were DCX positive, and the expression pattern of DCX was consistent with GFP (Supplementary Fig. S1A). However, few $\mathrm{GFP}^{+}$cells showed GFAP positive in the two groups (Supplementary Fig. S1B). This result was also confirmed by Confocal (Supplementary Fig. S1C). In addition, MAP2 was hardly expressed in NSCs most probably because it is a mature neuron dendritic marker (data were not shown).

Then, we tried to find out whether miR-29a overexpression influences the proliferation ability of NSCs. Cells were taken for immunostaining from the second day to the fourth day after transfection, respectively. Among them, cell proliferation marker Ki67 labeled proliferating cells and GFP indicated transfected cells. As a result, Ki67-positive cells were significantly decreased, from $45 \%$ on the second day to $<20 \%$ on the fourth day (Fig. 5C), suggesting that
NSCs were less active over culturing time. Moreover, the proportion of proliferating cells in the two groups showed no significant difference, indicating that overexpression of miR-29a did not affect the proliferation ability of NSCs (Fig. 5D).

\section{The expression level of miR-29a target ECM-related genes}

miR-29 targeted large number of ECM protein-encoding genes, including the collagen family, elastin, laminin, fibrillin, and so on, or genes related to ECM protein synthesis and degradation. Therefore, we selected several typical ECM genes to detect their expression level under miR-29 overexpression. We also tested Sox 2 and Nestin and the cell cycle-related gene $C d k 6$. Because the efficiency of electrotransfection was $\sim 30 \%-40 \%$, and low efficiency produced some interference with the quantification of RNA, we tried to use small molecule mimics to achieve the purpose of overexpression. As mentioned previously, miR29a-3p was more abundant in rat NSCs, so we synthesized miR-29a-3p small molecule mimics and transfected them into BMP-treated NSCs by Lipofectamine 3000 for RTqPCR assay.

It is very interesting that the expression level of ECMrelated genes, which miR-29 targeted, displayed different changes. Overexpression of miR-29a-3p significantly suppressed those ECM-related genes, including the collagen family gene Col3al (Fig. 6A), the laminin-encoding gene Lamc2 (Fig. 6B), the fibrillin family gene Fbnl (Fig. 6C), and Sparc (Fig. 6D), which encoded protein involved in ECM synthesis. Fstll (Fig. 6E), another interested gene also showed significant downregulation. But not all genes were downregulated. Col5a3 (Fig. 6F) showed no change in expression. Mmp24 was significantly increased (Fig. 6G). However, there was no significant difference in Sox 2 and Nestin between the miR-29a-3p overexpression and control (Fig. 6H). Moreover, Cdk6, an important regulator of cell cycle also showed no significant change after miR-29a-3p overexpression (Fig. 6I). These results indicated that miR29a overexpression maintained the characteristics and proliferative capacity of NSC cells, which is consistent with the results given in Fig. 5.

\section{Knockdown experiments of several typical miR-29a target ECM-related genes}

miR-29a overexpression altered the neurite outgrowth of NSCs, so we wanted to investigate whether downregulating certain genes may affect the neurite outgrowth. We selected three typical ECM-related genes-Fbn1, Fstl1, Lamc2. They were downregulated by miR-29 overexpression. Then we designed three specific siRNA for each of them to observe the effect of specific gene to NSC neurites. For morphological analysis, we stained DCX and GFAP as before.

First of all, we detected the efficiency of siRNA silencing (Fig. 7A). siRNA had different silencing efficiency compared with the control. For each gene, at least two siRNAs achieved $>80 \%$ silencing efficiency (Fig. 7A). We could see that DCX-positive cells were increased after siRNA transfection compared with the control (Fig. 7B). As shown previously, there were few GFAP-positive cells in the 


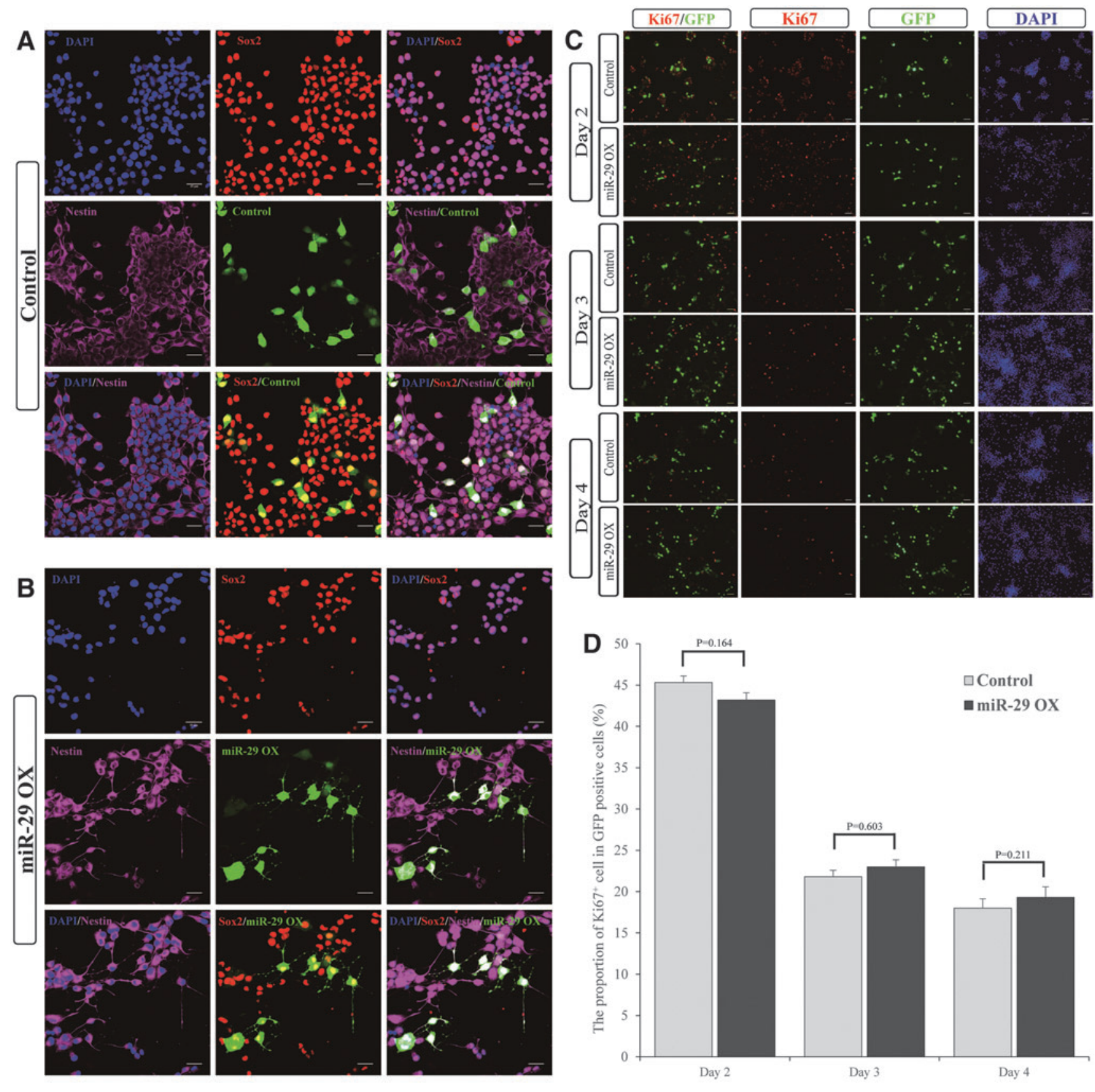

FIG. 5. Stem cell characteristics identification and proliferation analysis to NSCs. Cells transfected with control plasmid (A) and miR-29a overexpression plasmid (B) both expressed NSC markers Sox2 and Nestin. (C) Proliferation analysis of transfected NSCs over days. (D) The proportion of Ki67-positive cells in transfected NSCs. Scale bar: $25 \mu \mathrm{m}$ for $(\mathbf{A}, \mathbf{B})$; $50 \mu \mathrm{m}$ for (C). Data are given as mean $\pm \mathrm{SD}$.

control group. However, after siRNA transfection, there were varying increases of GFAP-positive cells and cell processes (Fig. 7C). The statistical results indicated that the most significant change was Fbnl-siRNA group, with a higher proportion of GFAP-positive cells in NSCs. In addition, proportion of GFAP-positive cells had also enhanced both in the Fstll-siRNA and Lamc2-siRNA groups compared with the control (Fig. 7D). In general, knockdown of certain ECM-related genes, which miR-29a targeted, might also achieve similar results with miR-29a overexpression in different degrees.

\section{RNA-Seq analysis of miR-29a regulated mRNAs in BMP-treated rat NSCs}

To identify potential mRNA targets of miR-29a in rat NSC cells, cells were transfected with miR-29a-3p mimics and control, followed by RNA-Seq of total RNA. Gene expression Venn diagram is given in Fig. 8A. We could see that 17,336 genes expressed in both groups, and 625 genes specifically expressed in miR-29a overexpression group, whereas only 754 genes expressed in the control group (Fig. 8A). FPKM values of the differential genes of each 


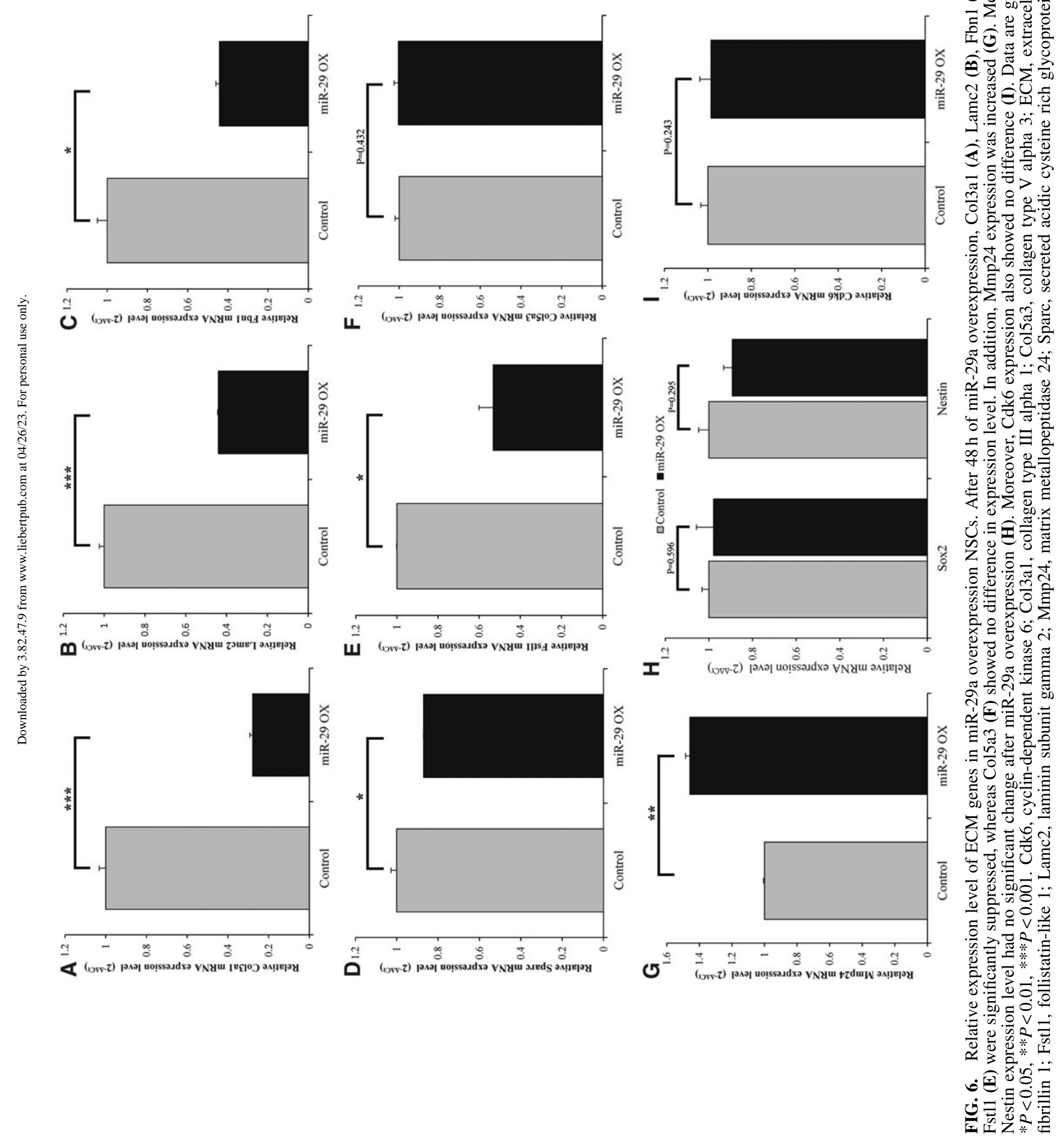




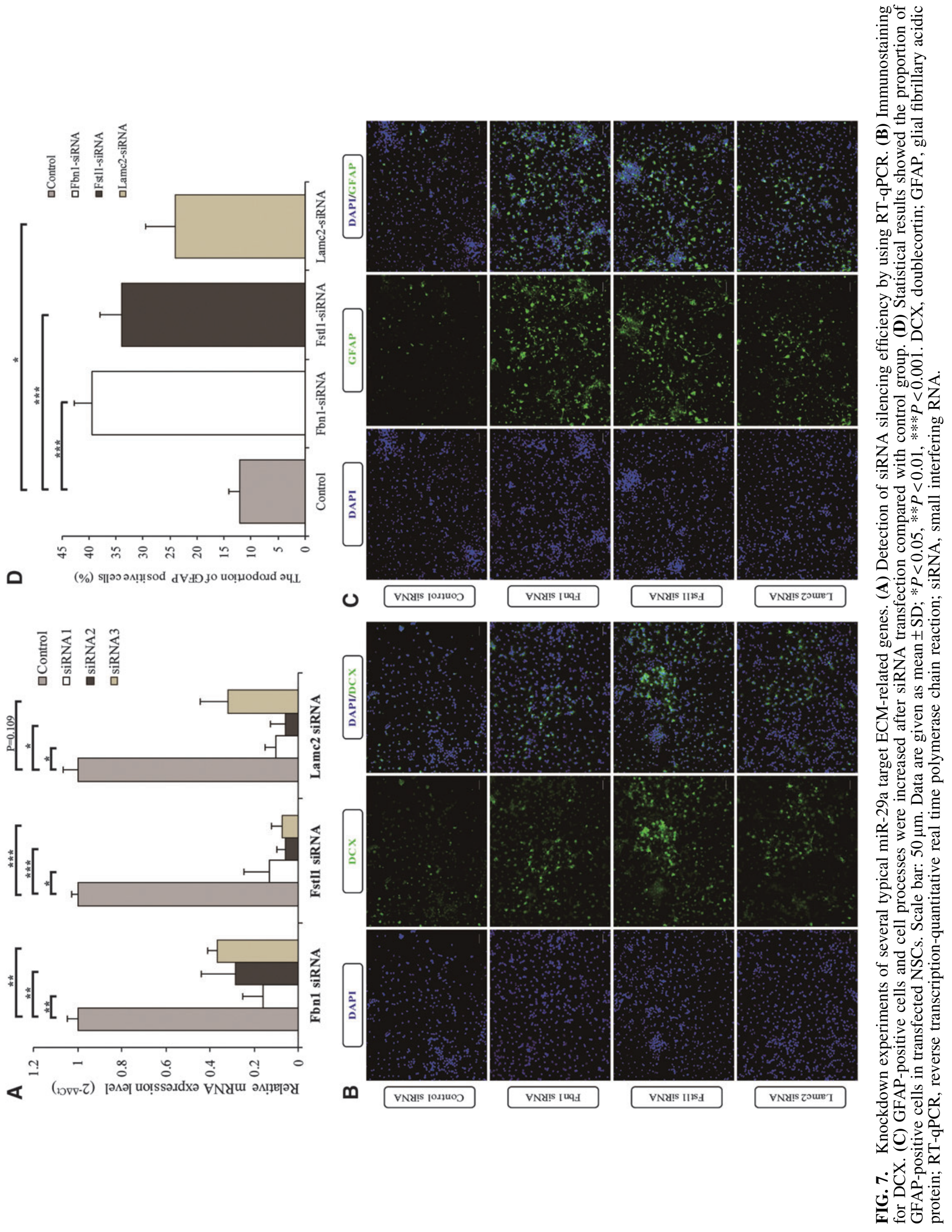


A

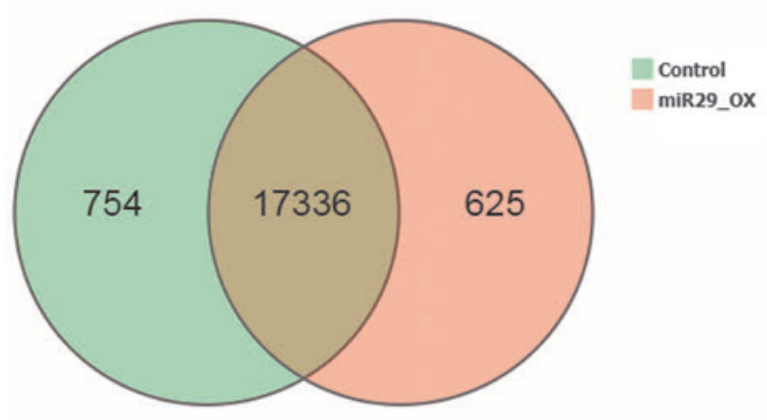

B

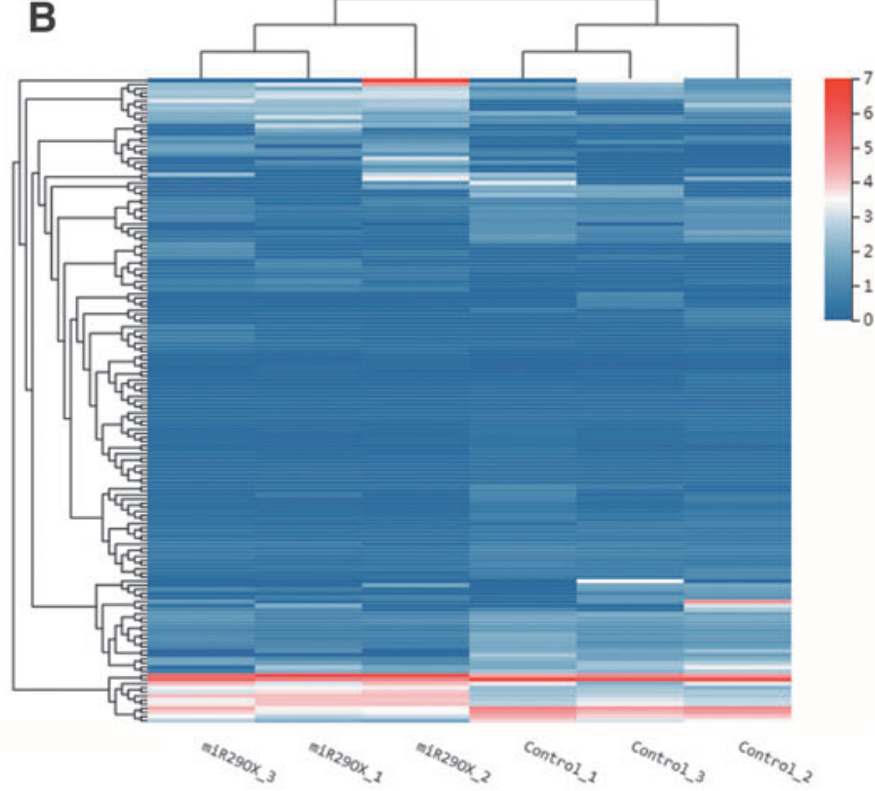

C

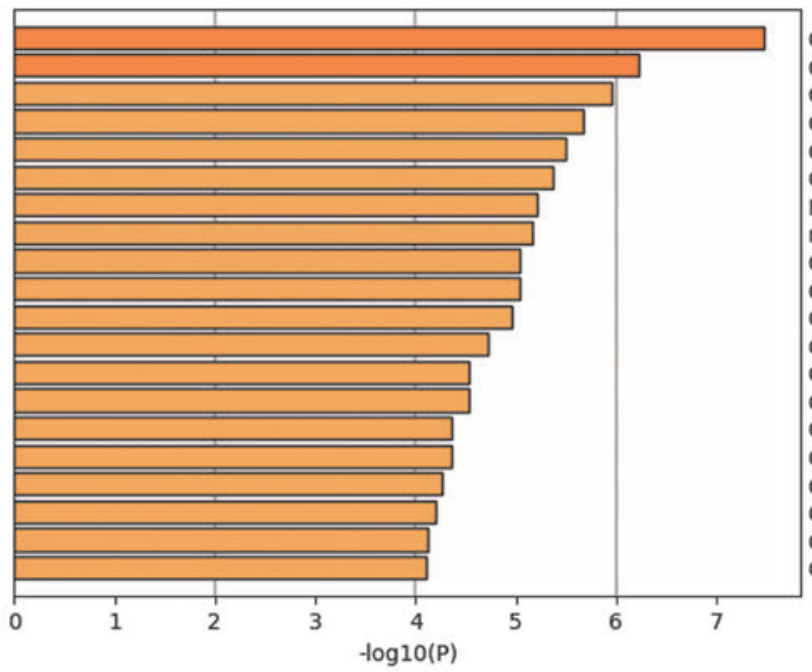

D

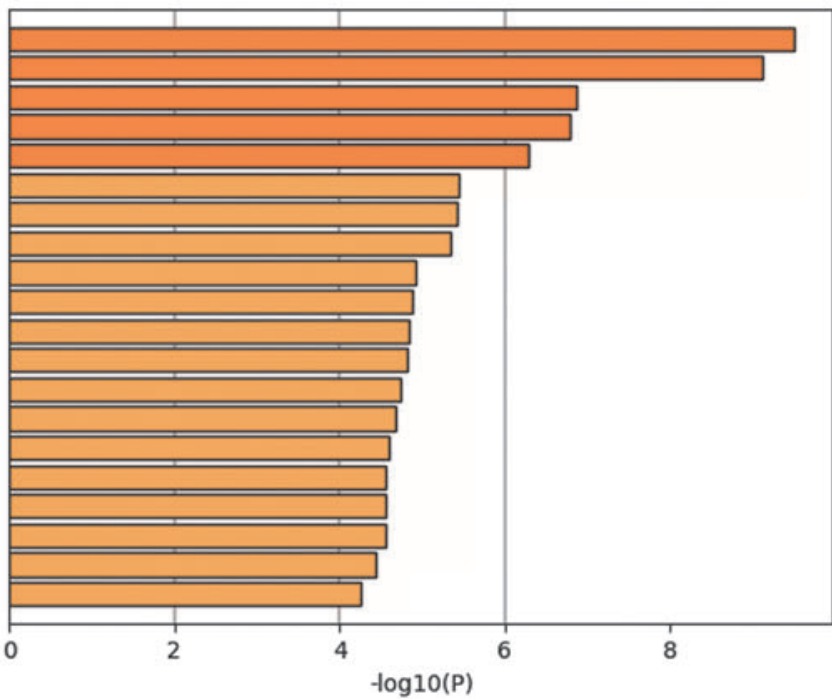

GO:0031589: cell-substrate adhesion

GO:0032101: regulation of response to external stimulus GO:0045785: positive regulation of cell adhesion GO:0043062: extracellular structure organization GO:0002062: chondrocyte differentiation GO:1901617: organic hydroxy compound biosynthetic process R-RNO-216083: Integrin cell surface interactions rno04512: ECM-receptor interaction GO:0048771: tissue remodeling

GO:0018958: phenol-containing compound metabolic process GO:0014065: phosphatidylinositol 3-kinase signaling GO:0001660: fever generation GO:0009611: response to wounding GO:0003012: muscle system process GO:0018108: peptidyl-tyrosine phosphorylation GO:0060445: branching involved in salivary gland morphogenesis GO:0030003: cellular cation homeostasis

GO:0098581: detection of external biotic stimulus GO:0048871: multicellular organismal homeostasis GO:0022600: digestive system process
GO:0061138: morphogenesis of a branching epithelium GO:0048608: reproductive structure development GO:0001568: blood vessel development R-RNO-373076: Class A/1 (Rhodopsin-like receptors) GO:0015711: organic anion transport GO:0030855: epithelial cell differentiation GO:0050900: leukocyte migration GO:0006270: DNA replication initiation GO:0070848: response to growth factor GO:0010035: response to inorganic substance GO:0043408: regulation of MAPK cascade GO:0050804: modulation of chemical synaptic transmission GO:0071774: response to fibroblast growth factor GO:0006954: inflammatory response GO:0048545: response to steroid hormone GO:0009266: response to temperature stimulus R-RNO-390696: Adrenoceptors GO:0015705: iodide transport GO:0060512: prostate gland morphogenesis GO:0001505: regulation of neurotransmitter levels

FIG. 8. KEGG Pathway and GO Biological Process Enrichment Analysis of RNA-Seq data. (A) Gene expression Venn diagram. (B) Differential gene expression clustering heat map. (C) Heatmap of enriched terms across downregulated genes, colored by $P$ values. (D) Heatmap of enriched terms across upregulated genes, colored by $P$ values. GO, gene ontology; RNA-Seq, RNA sequencing. 
comparison group were clustered, and the heat map is given in Fig. 8B. We calculated the FPKM values ratio of the control (numerator) to the miR-29a OX group (denominator), and filtered out genes with a value not $<1.5$, it can be considered as a specific downregulation. Conversely, if the ratio of the miR-29a OX (numerator) to the control (denominator) was not $<1.5$, it could be considered as a specific upregulation.

We analyzed the transcriptome data from RNA-seq to identify network processes, pathway maps, and GO (processes) between miR-29a versus control data sets. The top $20 \mathrm{GO}$ cellular processes downregulated or upregulated are given in Fig. 8C and D. Between two images, the gene clustering with specific downregulation was more interesting (Fig. 8C). Cell adhesion-related processes were downregulated such as cell-substrate adhesion, regulation of response to external stimulus, and positive regulation of cell adhesion (Fig. 8C). Branch morphology-related processes were upregulated like morphogenesis of a branching epithelium and reproductive structure development (Fig. 8D). These processes involve a large number of ECM synthesis and regulation. They may widely take part in intercellular communication, cell junction, cell migration, and intercellular signal transduction.

\section{Discussion}

In this study, we first identified the dynamic change of miR-29a after BMP-4 treatment and withdrew BMP-4 in rat NSCs. Then we found that there was a negative correlation between miR-29a expression and NSCs neurite outgrowth. We found that miR-29a overexpression increased the neurite number of NSCs significantly. Moreover, the complexity of neurites also increased, with multiple primary and secondary branches. Cell soma area of miR-29a overexpressed NSCs was dramatically reduced. Meanwhile, miR-29a overexpression did not alter the stem cell characteristics of NSCs. Finally, we also identified several ECM-related genes that downregulated under miR-29a-3p overexpression. Then siRNA were designed targeting three typical genes, Fbn 1, Fstl1, and Lamc2. Silencing any of Fbn1, Fstl1, and Lamc2 could promote neurite outgrowth of NSCs.

Neurogenesis is dynamically regulated by a number of extracellular signals including BMPs [57]. BMPs can act as short-range morphogens and profoundly affect adult NSC proliferation and differentiation [5,58]. BMP negatively regulates neurogenesis by promoting NSCs astroglial fate commitment and quiescence $[3,59,60]$. Certain levels of BMP-4 combination with FGF-2 reversibly induced the quiescent state of NSCs [3]. We introduced BMP-4 into NSCs, and were surprised to find the different morphology of neurites. We also found the role of miR-29 in neurite outgrowth.

TGF- $\beta$ /BMP signaling is involved in a vast majority of cellular processes and is fundamentally important throughout life. In addition, the crosstalk have been recognized between BMP/TGF- $\beta$ signaling and many other major signaling pathways such as MAPK, Wnt, Hedgehog, Notch, miR-29, and FGF $[61,62]$. TGF- $\beta 1$ is the effective target genes of miR-29 and meanwhile, miR-29b decreased the expression of TGF- $\beta 1$ [62]. These reports reveal the complex feedback between BMP and miR-29. However, the regulation network between them and the function in neurites still need further investigation. In this study, we only focused on the role of miR-29a in neurite outgrowth within the presence of BMP.

miR-29 family is associated with tumorigenesis, fibrosis, and ECM-related protein regulation [26,63]. Reports have shown that miR-29 family is upregulated in the adult cortex and striatum [64]. These findings indicate that miR-29 may play an unknown role in neural development and neurogenesis. In 2014, Shin et al. demonstrated that miR-29b plays a pivotal role in fetal mouse neurogenesis by regulating ICAT-mediated $\mathrm{Wnt} / \beta$-catenin signaling [65]. In 2018, Shi et al. showed that miR-29a promotes the neuronal differentiation and decreases the astrocytes differentiation of rat embryonic NSCs by targeting PTEN [66]. Our data show that overexpression of miR-29a neither changed the stem cell characteristics of adult rat hippocampus NSCs, nor affected its proliferation ability. These data suggested that miR-29a may have the different function as miR-29b in NSCs. In addition, the effects of miR-29a in adult and embryonic NSCs need more attention.

As a famous tumorigenesis regulator, the predicted target gene of miR-29 contains a large number of ECM-associated genes. miR-29a upregulation triggers axon branching by directly repressing DCX [46]. Zou et al. found that miR-29a can promote neurite outgrowth of PC12 cells by decreasing PTEN [48]. Another article also reported similar result. YAP-regulated miR-29a promotes the neurite outgrowth by decreasing PTEN in N2a cells [49]. These findings indicated that miR-29a is an important regulator of neurite outgrowth, and also suggest that miR-29a may be functional in NSCs and neurogenesis.

By analyzing the strand that is complementary to the RNA of interest, we focused on miR-29a-3p, which potentially targeted a large number of ECM genes. Our results also confirmed that miR-29a-3p is more abundant than $5 p$ in rat NSCs. We demonstrated that miR-29a overexpression significantly reduced the cell soma area and increased neurite outgrowth. Furthermore, neurites became more complex with multiple primary and secondary branches. RNA-Seq analysis of miR-29a-regulated mRNAs verified that the specific downregulation gene clusters are mostly related to cell adhesion, cell-substrate interaction, and extracellular structure. These genes may widely take part in intercellular communication, cell junction, cell migration, and intercellular signal transduction. This novel finding may be of benefit to study the interaction of NSCs and niche during development.

At the same time, we tried to knockdown miR-29a by using specific inhibitor (synthesized by GenePharma Co.). Previous reports point that brain-specific knockdown of miR-29 results in neuronal cell death in mice [67]. However, we had not yet observed the visible differences in rat NSCs (data not shown). One possible reason is that the expression level of miR-29a has already been downregulated in BMPtreated NSC cells (as shown in Fig. 2), so the inhibitor we used may not achieve any effect. Anyway, it needs further investigation.

During brain development and neurogenesis, the migration of newborn neural cells plays a pivotal role. Migration is controlled by a complex dynamic molecular regulatory network. Our findings provided a new sight that miRNAs 
might affect neural cells migration by regulating neurite outgrowth and branching. In addition, the function of miR29 in radial glial cells was also worth noting. As famous "scaffold" that guide neuronal migration, radial glial cells also serve as progenitors of neurons and glia [68-70]. Radial glial cells also help to direct axonal and dendritic process outgrowth, and then regulate synaptic development and function [68-70]. Does miR-29 function in radial glial cells? Whether it regulates the process of glial cells? What is the role of miR-29 in cell migration regulatory network? In future study, we will pay more attention to these questions and try to explore the intrinsic mechanism of miR-29 function.

\section{Conclusions}

During brain development and neurogenesis, the migration of new born neuronal cells plays an essential role and is critical to neocortex formation. The process of cell migration is also important to brain injury repair. These biological processes are partly controlled by neurite outgrowth and branching in NSCs. However, the regulatory mechanism is not sufficient and the functional study of miR-29 in NSC is limited. In this study, we investigated the function of miR29a in BMP-treated NSCs. First, we demonstrated that miR29a reduced cell soma area of rat NSCs and promoted neurite outgrowth and branching. Then, we found that NSCs of overexpressed miR-29a still maintain the stem cell characters. Finally, we identified that miR-29a regulated the neurite outgrowth of NSCs by downregulating the ECMrelated genes. This novel finding may provide a new insight for studying the interaction of NSCs and niche during migration, and also offered a possible theoretical basis to the migration mechanism of NSCs in brain development and brain damage repair.

\section{Author Disclosure Statement}

No competing financial interests exist.

\section{Funding Information}

The study was supported by National Natural Science Foundation of China grant (81901031), Natural Science Foundation of Shanghai (19ZR1445400), General Program Shanghai Municipal Health and Family Planning Commission grant (201740091), Natural Science Foundation of Shanghai (16ZR1430600), National Key R\&D Program of China (2017YFA0104100), National Natural Science Foundation of China grants $(31571058,31471029)$ and the National Major Scientific and Technological Special Project for Significant New Drugs Development (2018ZX09201002-005).

\section{Supplementary Material}

Supplementary Figure S1

\section{References}

1. Gage FH. (2000). Mammalian neural stem cells. Science 287:1433-1438.

2. Piatti VC and AF Schinder. (2018). Hippocampal mossy cells provide a fate switch for adult neural stem cells. Neuron 99:425-427.
3. Toda T and FH Gage. (2017). Review: adult neurogenesis contributes to hippocampal plasticity. Cell Tissue Res 373: 693-709.

4. Shi Y, X Zhao, J Hsieh, H Wichterle, S Impey, S Banerjee, $P$ Neveu and KS Kosik. (2010). MicroRNA regulation of neural stem cells and neurogenesis. J Neurosci 30:1493114936.

5. Goncalves JT, ST Schafer and FH Gage. (2016). Adult neurogenesis in the hippocampus: from stem cells to behavior. Cell 167:897-914.

6. Bartel DP. (2004). MicroRNAs: genomics, biogenesis, mechanism, and function. Cell 116:281-297.

7. Ambros V. (2004). The functions of animal microRNAs. Nature 431:350-355.

8. Ji F, X Lv and J Jiao. (2013). The role of microRNAs in neural stem cells and neurogenesis. J Genet Genomics 40: 61-66.

9. Shu SY, D Qing, B Wang, QY Zeng, YC Chen, Y Jin, CC Zeng and R Bao. (2013). Comparison of microRNA expression in hippocampus and the marginal division $(\mathrm{MrD})$ of the neostriatum in rats. J Biomed Sci 20:9.

10. Rajman M and G Schratt. (2017). MicroRNAs in neural development: from master regulators to fine-tuners. Development 144:2310-2322.

11. Zhao C, G Sun, S Li and Y Shi. (2009). A feedback regulatory loop involving microRNA-9 and nuclear receptor TLX in neural stem cell fate determination. Nat Struct Mol Biol 16:365-371.

12. Uchida N. (2010). MicroRNA-9 controls a migratory mechanism in human neural progenitor cells. Cell Stem Cell 6:294-296.

13. Lopez-Ramirez MA and S Nicoli. (2014). Role of miRNAs and epigenetics in neural stem cell fate determination. Epigenetics 9:90-100.

14. Waites CL, AM Craig and CC Garner. (2005). Mechanisms of vertebrate synaptogenesis. Annu Rev Neurosci 28:251274.

15. Webb SJ, CS Monk and CA Nelson. (2001). Mechanisms of postnatal neurobiological development: implications for human development. Dev Neuropsychol 19:147-171.

16. Smrt RD, KE Szulwach, RL Pfeiffer, X Li, W Guo, M Pathania, ZQ Teng, Y Luo, J Peng, et al. (2010). MicroRNA miR-137 regulates neuronal maturation by targeting ubiquitin ligase mind bomb-1. Stem Cells 28:1060-1070.

17. Dajas-Bailador F, B Bonev, P Garcez, P Stanley, F Guillemot and N Papalopulu. (2012). microRNA-9 regulates axon extension and branching by targeting Map1b in mouse cortical neurons. Nat Neurosci 15:697-699.

18. Strickland IT, L Richards, FE Holmes, D Wynick, JB Uney and LF Wong. (2011). Axotomy-induced miR-21 promotes axon growth in adult dorsal root ganglion neurons. PLoS One 6:e23423.

19. Wu D and AK Murashov. (2013). MicroRNA-431 regulates axon regeneration in mature sensory neurons by targeting the Wnt antagonist Kremen1. Front Mol Neurosci 6:35.

20. Zhang J, J Zhang, LH Liu, Y Zhou, YP Li, ZH Shao, YJ Wu, MJ Li, YY Fan and HJ Shi. (2011). Effects of miR-541 on neurite outgrowth during neuronal differentiation. Cell Biochem Funct 29:279-286.

21. Wayman GA, M Davare, H Ando, D Fortin, O Varlamova, HY Cheng, D Marks, K Obrietan, TR Soderling, RH Goodman and S Impey. (2008). An activity-regulated microRNA controls dendritic plasticity by down-regulating p250GAP. Proc Natl Acad Sci U S A 105:9093-9098. 
22. Thiriet M. (2011). Extracellular matrix. In: Cell and Tissue Organization in the Circulatory and Ventilatory Systems. Mecham RP, ed. Springer, New York, pp 485-519.

23. Theocharis AD, SS Skandalis, C Gialeli and NK Karamanos. (2016). Extracellular matrix structure. Adv Drug Deliv Rev 97:4-27.

24. Boon RA, T Seeger, S Heydt, A Fischer, E Hergenreider, AJ Horrevoets, M Vinciguerra, N Rosenthal, S Sciacca, et al. (2011). MicroRNA-29 in aortic dilation: implications for aneurysm formation. Circ Res 109:1115-1119.

25. Hubmacher D and SS Apte. (2013). The biology of the extracellular matrix: novel insights. Curr Opin Rheumatol 25:65-70.

26. Kriegel AJ, Y Liu, Y Fang, X Ding and M Liang. (2012). The miR-29 family: genomics, cell biology, and relevance to renal and cardiovascular injury. Physiol Genomics 44: 237-244.

27. Qiu F, R Sun, N Deng, T Guo, Y Cao, Y Yu, X Wang, B Zou, S Zhang, et al. (2015). miR-29a/b enhances cell migration and invasion in nasopharyngeal carcinoma progression by regulating SPARC and COL3A1 gene expression. PLoS One 10:e0120969.

28. Merk DR, JT Chin, BA Dake, L Maegdefessel, MO Miller, N Kimura, PS Tsao, C Iosef, GJ Berry, et al. (2012). miR$29 \mathrm{~b}$ participates in early aneurysm development in Marfan syndrome. Circ Res 110:312-324.

29. Maegdefessel L, J Azuma, R Toh, DR Merk, A Deng, JT Chin, U Raaz, AM Schoelmerich, A Raiesdana, et al. (2012). Inhibition of microRNA-29b reduces murine abdominal aortic aneurysm development. J Clin Invest 122: 497-506.

30. Cushing L, PP Kuang, J Qian, F Shao, J Wu, F Little, VJ Thannickal, WV Cardoso and Lü J. (2011). miR-29 is a major regulator of genes associated with pulmonary fibrosis. Am J Respir Cell Mol Biol 45:287-294.

31. Zhang P, A Huang, J Ferruzzi, RP Mecham, BC Starcher, G Tellides, JD Humphrey, FJ Giordano, LE Niklason and WC Sessa. (2012). Inhibition of microRNA-29 enhances elastin levels in cells haploinsufficient for elastin and in bioengineered vessels-brief report. Arterioscler Thromb Vasc Biol 32:756-759.

32. Fabbri M, R Garzon, A Cimmino, Z Liu, N Zanesi, E Callegari, S Liu, H Alder, S Costinean, et al. (2007). MicroRNA-29 family reverts aberrant methylation in lung cancer by targeting DNA methyltransferases $3 \mathrm{~A}$ and $3 \mathrm{~B}$. Proc Natl Acad Sci U S A 104:15805-15810.

33. Mahati S, L Xiao, Y Yang, R Mao and Y Bao. (2017). miR29a suppresses growth and migration of hepatocellular carcinoma by regulating CLDN1. Biochem Biophys Res Commun 486:732-737.

34. Trãhoux S, F Lahdaoui, Y Delpu, F Renaud, E Leteurtre, J Torrisani, N Jonckheere and I Van Seuningen. (2015). Micro-RNAs miR-29a and miR-330-5p function as tumor suppressors by targeting the MUC1 mucin in pancreatic cancer cells. Biochim Biophys Acta 1853:2392-2403.

35. Zhu C, Y Wang, W Kuai, X Sun, H Chen and Z Hong. (2013). Prognostic value of miR-29a expression in pediatric acute myeloid leukemia. Clin Biochem 46:49-53.

36. Li R, J Liu, Q Li, G Chen and X Yu. (2016). miR-29a suppresses growth and metastasis in papillary thyroid carcinoma by targeting AKT3. Tumour Biol 37:3987-3996.

37. Stamato MA, G Juli, E Romeo, D Ronchetti, M Arbitrio, D Caracciolo, A Neri, P Tagliaferri, P Tassone and N Amodio. (2017). Inhibition of EZH2 triggers the tumor sup- pressive miR-29b network in multiple myeloma. Oncotarget 8:106527-106537.

38. van Rooij E, LB Sutherland, JE Thatcher, JM DiMaio, RH Naseem, WS Marshall, JA Hill and EN Olson. (2008). Dysregulation of microRNAs after myocardial infarction reveals a role of miR-29 in cardiac fibrosis. Proc Natl Acad Sci U S A 105:13027-13032.

39. Lu L, X Xue, J Lan, Y Gao, Z Xiong, H Zhang, W Jiang, W Song and Q Zhi. (2014). MicroRNA-29a upregulates MMP2 in oral squamous cell carcinoma to promote cancer invasion and anti-apoptosis. Biomed Pharmacother 68:1319.

40. Park SY, JH Lee, M Ha, JW Nam and VN Kim. (2009). miR-29 miRNAs activate p53 by targeting p85 alpha and CDC42. Nat Struct Mol Biol 16:23-29.

41. Qin RH, H Tao, SH Ni, P Shi, C Dai and KH Shi. (2018). microRNA-29a inhibits cardiac fibrosis in Sprague-Dawley rats by downregulating the expression of DNMT3A. Anatol J Cardiol 20:198-205.

42. Tao H, ZW Chen, JJ Yang and KH Shi. (2016). MicroRNA-29a suppresses cardiac fibroblasts proliferation via targeting VEGF-A/MAPK signal pathway. Int J Biol Macromol 88:414-423.

43. Huang YH, HC Kuo, YL Yang and FS Wang. (2019). MicroRNA-29a is a key regulon that regulates BRD4 and mitigates liver fibrosis in mice by inhibiting hepatic stellate cell activation. Int J Med Sci 16:212-220.

44. Yang YL, FS Wang, SC Li, MM Tiao and YH Huang. (2017). MicroRNA-29a alleviates bile duct ligation exacerbation of hepatic fibrosis in mice through epigenetic control of methyltransferases. Int J Mol Sci 18:192.

45. Majer A, SJ Medina, Y Niu, B Abrenica, KJ Manguiat, KL Frost, CS Philipson, DL Sorensen and SA Booth. (2012). Early mechanisms of pathobiology are revealed by transcriptional temporal dynamics in hippocampal CA1 neurons of prion infected mice. PLoS Pathog 8:e1003002.

46. Li H, S Mao, H Wang, K Zen, C Zhang and L Li. (2014). MicroRNA-29a modulates axon branching by targeting doublecortin in primary neurons. Protein Cell 5:160-169.

47. Cohen D, M Segal and O Reiner. (2008). Doublecortin supports the development of dendritic arbors in primary hippocampal neurons. Dev Neurosci 30:187-199.

48. Zou H, Y Ding, K Wang, E Xiong, W Peng, F Du, Z Zhang, J Liu and A Gong. (2015). MicroRNA-29A/PTEN pathway modulates neurite outgrowth in PC12 cells. Neuroscience 291:289-300.

49. Tan C, C Yu, Z Song, H Zou, X Xu and J Liu. (2017). Expression of microRNA-29a regulated by Yes-associated protein modulates the neurite outgrowth in N2a cells. Biomed Res Int 2017:5251236.

50. Kim D, B Langmead and SL Salzberg. (2015). HISAT: a fast spliced aligner with low memory requirements. Nat Methods 12:357-360.

51. Langmead B and SL Salzberg. (2012). Fast gapped-read alignment with Bowtie 2. Nat Methods 9:357-359.

52. Li B and CN Dewey. (2011). RSEM: accurate transcript quantification from RNA-Seq data with or without a reference genome. BMC Bioinformatics 12:323.

53. Sholl DA. (1953). Dendritic organization of the neurons in the visual and motor cortices of the cat. J Anat 87:387-406.

54. Ferreira TA, LL Iacono and CT Gross. (2010). Serotonin receptor $1 \mathrm{~A}$ modulates actin dynamics and restricts dendritic growth in hippocampal neurons. Eur J Neurosci 32: $18-26$. 
55. Cushing L, P Kuang and J Lu. (2015). The role of miR-29 in pulmonary fibrosis. Biochem Cell Biol 93:109-118.

56. Kutzing MK, CG Langhammer, V Luo, H Lakdawala and BL Firestein. (2010). Automated Sholl analysis of digitized neuronal morphology at multiple scales. J Vis Exp 14:pii:2354.

57. Armenteros $T, Z$ Andreu, $R$ Hortiguela, DC Lie and $H$ Mira. (2018). BMP and WNT signalling cooperate through LEF1 in the neuronal specification of adult hippocampal neural stem and progenitor cells. Sci Rep 8:9241.

58. Choe Y, SJ Pleasure and H Mira. (2015). Control of adult neurogenesis by short-range morphogenic-signaling molecules. Cold Spring Harb Perspect Biol 8:a018887.

59. Lim DA, AD Tramontin, JM Trevejo, DG Herrera, JM Garcia-Verdugo and A Alvarez-Buylla. (2000). Noggin antagonizes BMP signaling to create a niche for adult neurogenesis. Neuron 28:713-726.

60. Mira H, Z Andreu, H Suh, DC Lie, S Jessberger, A Consiglio, J San Emeterio, R Hortiguela, MA MarquesTorrejon, et al. (2010). Signaling through BMPR-IA regulates quiescence and long-term activity of neural stem cells in the adult hippocampus. Cell Stem Cell 7:78-89.

61. Chen G, C Deng and YP Li. (2012). TGF- $\beta$ and BMP signaling in osteoblast differentiation and bone formation. Int J Biol Sci 8:272-288.

62. Luna C, G Li, Qiu, DL Epstein and P Gonzalez. (2011). Cross-talk between miR-29 and transforming growth factor-betas in trabecular meshwork cells. Invest Ophthalmol Vis Sci 52:3567-3572.

63. Deng Z, Y He, X Yang, H Shi, A Shi, L Lu and L He. (2017). MicroRNA-29: a crucial player in fibrotic disease. Mol Diagn Ther 21:285-294.

64. Landgraf P, M Rusu, R Sheridan, A Sewer, N Iovino, A Aravin, S Pfeffer, A Rice, AO Kamphorst, et al. (2007). A mammalian microRNA expression atlas based on small RNA library sequencing. Cell 129:1401-1414.

65. Shin J, Y Shin, SM Oh, H Yang, WJ Yu, JP Lee, SO Huh, SH Lee, YH Suh, S Chung and HS Kim. (2014). MiR-29b controls fetal mouse neurogenesis by regulating ICATmediated Wnt/beta-catenin signaling. Cell Death Dis 5: e1473.
66. Shi Z, H Zhou, L Lu, B Pan, Z Wei, J Liu, J Li, S Yuan, Y Kang, et al. (2018). MicroRNA-29a regulates neural stem cell neuronal differentiation by targeting PTEN. J Cell Biochem 119:5813-5820.

67. Roshan R, S Shridhar, MA Sarangdhar, A Banik, M Chawla, M Garg, VP Singh and B Pillai. (2014). Brainspecific knockdown of miR-29 results in neuronal cell death and ataxia in mice. RNA 20:1287-1297.

68. Rakic P. (1972). Mode of cell migration to the superficial layers of fetal monkey neocortex. J Comp Neurol 145:61-83.

69. Noctor SC, AC Flint, TA Weissman, RS Dammerman and AR Kriegstein. (2001). Neurons derived from radial glial cells establish radial units in neocortex. Nature 409:714-720.

70. Sild M and ES Ruthazer. (2011). Radial glia: progenitor, pathway, and partner. Neuroscientist 17:288-302.

Address correspondence to:

Dr. Xinxin Han

Oral Biomedical Engineering Laboratory Shanghai Stomatological Hospital Fudan University

No. 356, Beijing East Road, Huangpu District Shanghai 200001

China

E-mail: xxhan@fudan.edu.cn

Dr. Jun $X u$

East Hospital

Tongji University School of Medicine No. 150, Jimo Road, Pudong District

Shanghai 200120

China

E-mail: xunymc2000@yahoo.com

Received for publication August 10, 2019

Accepted after revision December 27, 2019

Prepublished on Liebert Instant Online December 28, 2019 